\title{
The Downs and Ups of Mechanistic Research: Circadian Rhythm Research as an Exemplar
}

\author{
William Bechtel
}

Received: 9 June 2010/ Accepted: 9 June 2010/Published online: 3 September 2010

(C) The Author(s) 2010. This article is published with open access at Springerlink.com

\begin{abstract}
In the context of mechanistic explanation, reductionistic research pursues a decomposition of complex systems into their component parts and operations. Using research on the mechanisms responsible for circadian rhythms, I consider both the gains that have been made by discovering genes and proteins that figure in these intracellular oscillators and also highlight the increasingly recognized need to understand higher-level integration, both between cells in the central oscillator and between the central and peripheral oscillators. This history illustrates a common need to complement reductionistic inquiry with investigations at higher-levels. Unlike most other accounts of reduction, the mechanistic framework accommodates this complementary relationship between reductionistic and systems approaches.
\end{abstract}

\section{Introduction}

Philosophers have tended to discuss reduction in terms of a relation between theories in which the reduced theory could be derived form the reducing theory (Nagel 1961; for discussion of theory reduction and the alternatives, see Bechtel and Hamilton 2007). Life scientists, however, seldom present theories as systematic sets of propositions or concern themselves with logical relations between theories. Rather, they typically try to explain a phenomenon by identifying and describing the mechanism responsible for it (for representative discussions of mechanistic explanation, see Bechtel and Richardson 1993; Bechtel 2008; Machamer et al. 2000; Thagard 2006). The quest for mechanistic explanations, though, brings with it its own version of reductionism-since a mechanism involves parts, each

\footnotetext{
W. Bechtel $(\bowtie)$

Department of Philosophy, Center for Chronobiology, and Interdisciplinary Programs in Science Studies and Cognitive Science, University of California, San Diego, CA, USA e-mail: bill@mechnism.ucsd.edu
} 
performing operations, organized so as to generate the phenomenon to be explained (under appropriate contextual conditions), a premium is placed on decomposing the mechanism into its parts and operations. Parts and operations are at a lower level of organization than the mechanism as a whole, and pursuit of inquiry at this level is commonly characterized as reductionistic by scientists.

After successfully decomposing a mechanism into its parts, a researcher may desire to understand how a given part performs the operation associated with it. Typically, this will require iterating the initial procedure, now treating the part as the mechanism of interest and decomposing it into its parts (sub-parts of the initial mechanism) and identifying its operations (the operations in virtue of which the part of the initial mechanism performs its operation). Although there is no principled point at which to terminate this process of iterative decomposition, individual researchers typically lack the instruments and techniques to conduct more than one or two rounds of decomposition. Other researchers may come along to take up the project of explaining the parts that the researcher took to be the basic units in her explanation. But at some point relevant specialists recognize that further decomposition does not contribute to explaining the initial phenomenon of interest. In the life sciences, explanation typically bottoms out at the level of molecules-genes and proteins. Accordingly, in biology, the most extreme reductionists tend to be biochemists and molecular biologists, who pursue explanations in terms of genes, proteins, and the molecules that figure in biochemical reactions. For some biologists, as well as their philosophical defenders (Bickle 2003; Rosenberg 2006), a phenomenon is only really explained by an account at that level.

Very often, though, the discovery of the operations performed by the parts of a mechanism is accompanied by the recognition that these operations are affected by the operations of other parts. The result is that researchers must contextualize the account of particular parts and operations within a larger system in order to understand how they contribute to the phenomenon of interest. This process of contextualizing components too is iterated-systems built out of components are themselves components of yet higher-level systems. It is particularly important to take systemic organization into account when the system contains feedback loops whereby operations later in what is treated as a sequence affect the performance of earlier operations. The result is that researchers must complement the reductionistic focus on parts and operations with attempts to understand the consequences of spatial and temporal organization both within a mechanism at a given level and between the mechanism and its environment at a higher level.

I will illustrate both the path down to the molecular parts of a mechanism and the path back up to the systems level with recent research on circadian rhythms. I begin with the first step in any mechanistic research-delineating the phenomenon under investigation. This will also serve to familiarize those not familiar with circadian rhythms with the explanatory challenges researchers confronted. I will then describe two stages of reductionistic research on circadian rhythms: (1) the identification of a set of cells thought to be uniquely responsible for maintaining rhythms (the central oscillator) and (2) the discovery of the parts and operations within these cells that explained their oscillatory behavior. I then consider two stages 
in the upward path: (1) conceptualizing and modeling the integrated network of reactions within individual cells, and (2) discovering the relations between cells within the central oscillator and between it and the entrainment system and peripheral oscillators that are more directly involved in generating circadian behavior.

\section{Delineating the Phenomenon}

Before researchers can begin to identify a mechanism, they need to characterize the phenomenon to be explained. Philosophical accounts often miss the important challenges confronted in this endeavor by construing science as simply explaining observations that can be made by opening one's eyes and reporting what one observes. Few phenomena in biology are of this sort and very often a great deal of investigation is required to specify the phenomenon, including experiments using complex techniques to intervene in nature. A further complication is that it is not infrequent that as research on the responsible mechanism progresses, researchers revise their understanding of the phenomenon.

Although humans often assume that, except for the obvious need for sleep, our physiology and behavior (including our capacities to process information) are the same at different times during the day, many features of our physiology and behavior are modulated over a $24 \mathrm{~h}$ cycle. This is true not just in humans but in organisms ranging from cyanobacteria to plants, fungi, and other animals. Some of these cycles were recognized by the ancient Greeks: Androsthenes of Thasus, a captain in Alexander's fleet, recorded the daily movement of the leaves of the tamarind tree, while Hippocrates and Galen both observed how temperature in patients with fevers varied with time of day. But circadian phenomena are characterized by more than daily cycles. Circadian cycles are controlled endogenously, a feature that can only be established by showing that the cycles continue when organisms are kept in constant conditions. One clue that the cycles are endogenously controlled is that they often depart somewhat from a precise $24 \mathrm{~h}$ rhythm (hence, circa-about + dies-day).

The variability in internally generated rhythms alerted researchers to the fact that, like an old-fashioned watch, whatever produced endogenous circadian rhythms (referred to as a clock by analogy to the clocks humans engineer to maintain social timekeeping) required regular resetting from an external source such as the rising or setting of the sun (referred to as a Zeitgeber). Such entrainment to Zeitgebers is crucial in organisms that need to adjust to seasonal variations in periods of daylight or to relocation to different time zones. Yet another critical feature of circadian phenomena is that they are relatively unaffected by temperature. This presented a significant explanatory challenge since biochemical reactions proceed more rapidly at higher temperatures (typically doubling in rate for every $10^{\circ} \mathrm{C}$ increase). The circadian phenomenon to be explained accordingly involved (1) endogenous generation of approximately $24 \mathrm{~h}$ rhythms that (2) are entrainable by light or other Zeitgebers and (3) are temperature compensated. 


\section{The Downward Path I: Direct Localization of the Circadian Clock}

A common strategy in mechanistic research is to seek a component within the system that exhibits a phenomenon and can be considered responsible for it (Bechtel and Richardson 1993, chapter 4). Carl Richter initiated such a search for the clock in mammals, lesioning various organs such as adrenals, gonads, pituitary, thyroid, pineal, and pancreas in blind rats in the attempt to impair the rhythm when no Zeitgebers were available. Finally, he determined that lesions to the front part of the hypothalamus eliminated rhythmic behaviors (Richter 1967). In the early 1970s two investigators identified the suprachiasmatic nucleus $(\mathrm{SCN})$, a structure in the anterior hypothalamus consisting of approximately 16,000-20,000 cells $(8,000-10,000$ on each side of the brain), as the locus of the circadian clock in mammals: Stephan and Zucker (1972) showed that lesions to the SCN produced deficits in nocturnal locomotion and drinking behavior, while Moore and Eichler (1972) demonstrated that lesions to the SCN eliminated hormonal cycling in rats. Moore (1973) also employed ${ }^{3} \mathrm{H}$-amino acids tracers to identify a pathway from the eyes to the SCN, thereby showing that there was a route through which light information could be supplied to the SCN to entrain it. A few years later, Inouye and Kawamura (1979) recorded electrical activity from the SCN and revealed that even when inputs were destroyed, electrical activity in the SCN continued to exhibit circadian rhythms. These various strands of evidence indicated that the SCN was or contained the clock but Ralph et al. (1990) performed the decisive experiment. They transplanted the SCN from a mutant golden hamster (known as the tau mutant) with significantly shortened circadian rhythms (20.2 h) into a host that had been rendered arrhythmic after its SCN was removed. The recipient exhibited a rhythm with a period of approximately $20.2 \mathrm{~h}$, which could only be attributed to the transplanted SCN.

In comparable research conducted on Drosophila (fruit flies) during this period a small collection of lateral neurons were found to sustain oscillations. This, plus the fact that circadian phenomena are also exhibited in single cell organisms, prompted investigators to ask whether individual neurons in the SCN could keep time. Welsh et al. (1995) developed procedures for culturing and recording from individual mouse SCN cells and demonstrated that individual cells could sustain rhythms. Despite the advance in knowledge provided by the determination that individual cells in the SCN exhibited circadian rhythms, little explanatory gain was realized since the research did not reveal how the rhythms were generated. It simply revealed the locus of the clock and prepared for the second stage of the downward path.

\section{The Downward Path II: Identifying the Clockworks}

Mechanistic research requires more than identifying where in the organism a given phenomenon is produced-it also requires showing how components that on their own do not exhibit the phenomena work together to generate it (Bechtel and Richardson 1993, chapter 6). In research conducted simultaneously with the discovery of the SCN in mammals, Konopka and Benzer (1971), working with 
Drosophila, identified a gene, period or per, with three mutant forms that resulted in flies that either exhibited rhythms longer or shorter than $24 \mathrm{~h}$ or were arrhythmic. Like other genes, per is transcribed into mRNA and then translated into a protein, PER. (Gene and protein names are customarily abbreviated, typically in three letters, with gene names in italics - all in lower case in Drosophila but with the first letter capitalized in mammals - and proteins names in capital letters.) The differentiation of a gene, mRNA, and protein represented a first step in decomposing the clock into multiple working parts. But what does PER do? In the 1980s researchers explored the possibility that PER was involved in activities at the cell membrane that affected communication between cells, but this bore little fruit. The same research that localized per expression to the lateral neurons also revealed that concentrations of PER and its mRNA oscillated (Liu et al. 1988). In wildtype flies PER peaked early at night and was undetectable by mid-day (it peaked early or late, or was not detected at all, in mutants). Moreover, when per mRNA was measured in isolated fly heads, it too was rhythmic, with a peak 4-6 h earlier than the peak for PER. Rosbash and his collaborators soon advanced a model (Fig. 1) according to which PER feeds back onto per transcription to inhibit its expression (Hardin et al. 1990). This model received support from the observation that PER accumulated in the nucleus prior to its disappearance. This model suggested an explanation for the renewal of the cycle: after PER inhibits per transcription, it begins to break down, and when levels of PER drop sufficiently, per is released from inhibition and transcription begins again.

This model, however, confronted a serious problem. For a protein to bind with DNA so as to inhibit its transcription, a region or domain that binds to DNA is needed, but PER lacked such a domain. Thus, PER could not directly regulate per transcription. PER did possess another region that it shared with two other proteins - the mammalian aryl hydrocarbon receptor (ARNT) and the fly singleminded (SIM) protein - that were thought to be transcription regulating proteins. This region, known as the PAS domain after these three proteins in which it was first found, permits the protein to bind with another protein to create a kind of compound known as a dimer. Huang et al. (1993) hypothesized that PER acted by forming a dimer with an unknown transcription factor, prompting a search for such a protein. By pursuing a similar strategy as Konopka and searching for additional mutants that

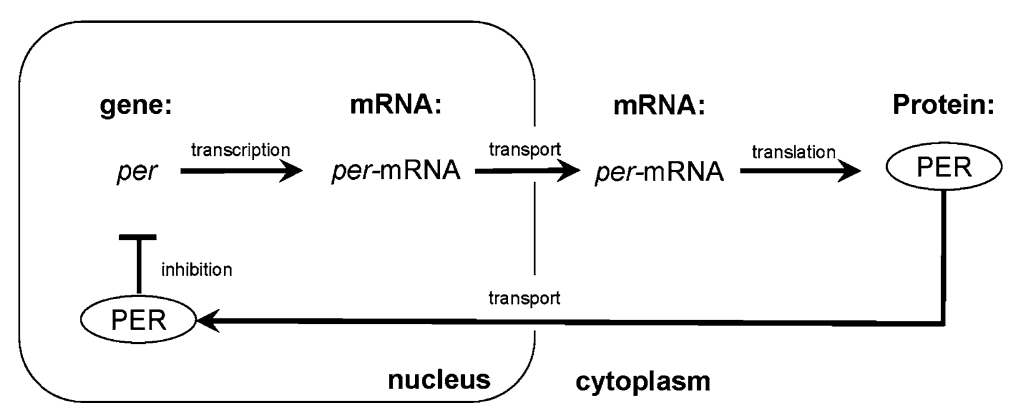

Fig. 1 Model of role of per in mechanism of circadian oscillation 
showed altered rhythms, Sehgal et al. (1994) discovered a second clock gene, timeless (tim), whose mutants manifested no rhythmic behavior. In a follow up paper, they showed that in $\operatorname{tim}^{O}$ mutants PER failed to enter the nucleus and advanced evidence that without TIM, PER was unstable in the cytoplasm (Vosshall et al. 1994). Saez and Young (1996) discovered how PER and TIM interacted and entered the nucleus-both proteins contain a cytoplasmic localization domain (CLD) that, if exposed, prevents the protein from entering the nucleus. The formation of the PER:TIM dimer, which is established through binding at two different sites, blocks the CLD domain, allowing the dimer to enter the nucleus.

Although the discovery of tim revealed an unexpected part of the mechanism, it did not satisfy Huang et al.'s hypothesis, since it too lacked a domain needed to bind with DNA and inhibit the transcription of per (or tim). The discovery of how inhibition was accomplished resulted from an independent line of research on mammalian circadian rhythms for which, up to that time, no clock genes had been discovered. To find mammalian clock genes, Takahashi and Pinto proceeded (again, much like Konopka and Sehgal et al.) by exposing mice to mutagens and screening offspring for circadian abnormalities. They created a mutant with a long circadian rhythm and named the responsible gene Clock (for Circadian Locomotor Output Cycles Kaput). Homozygotic mutants became arrhythmic after several days in constant darkness (Vitaterna et al. 1994). Clock was found to have the requisite domain to bind to DNA and serve as a direct transcription factor. It also possessed a PAS region and so could form a dimer. Once the Clock gene had been cloned and sequenced, Steve Kay and his collaborators found a homologue in Drosophila, dclock (Darlington et al. 1998). They determined that dclock mRNA cycles out of phase with per or tim, suggesting that CLOCK (together with its dimerization partner BMAL1) stimulated per and tim transcription when PER and TIM were in low concentrations in the nucleus. Concentrations of CLOCK were reduced when PER and TIM concentrations in the nucleus increased. They concluded that dCLOCK "closes the feedback loop" and offered the following account of the operation of the oscillator:

A dCLOCK-dBMAL1 complex drives expression of per and tim by binding an E-box that is present in their promoters. With time, PER and TIM heterodimers accumulate, translocate to the nucleus, and act as dominant negative inhibitors of dCLOCK-dBMAL1. As mRNA and protein levels fall, the inhibition is relieved, which allows dCLOCK-dBMAL1 to initiate a new round of synthesis... It is tempting to speculate that the Drosophila four-component transcriptional feedback loop described here is sufficient to generate a rudimentary circadian rhythm. This oscillation would be amplified by other, unknown proteins that regulate RNA stability, protein stability, and phosphorylation of the oscillator components. (Darlington et al. 1998, p. 1602).

In short order yet other components of the Drosophila clock were discovered. One of these, DOUBLETIME (DBT), is a protein kinase that attaches a phosphate to PER, marking it for degradation by the ubiquitin/proteasome pathway in the cell (Price et al. 1998). The degradation of PER continues until sufficient TIM is produced to dimerize with it; this partially explains the lag in transfer of PER to the 
nucleus. In addition to binding with PER and facilitating its transfer into the nucleus, TIM figures in the process of entraining the clock in Drosophila since it is broken down when the fly is exposed to light (Hunter-Ensor et al. 1996). Although light affects TIM concentrations at any time, the reduced concentrations of TIM have greatest effect on circadian oscillations when light pulses are administered in the early night (when it delays the accumulation of TIM needed to dimerize with PER and facilitate its transport into the nucleus) and in the late night (when it expedites the breakdown of the PER:TIM dimer and stops its inhibitory effects on per and tim synthesis). Hunter-Ensor et al. were unable to identify the agent involved in the breakdown of TIM, but evidence that circadian rhythms in Drosophila were most affected by blue light led Emery et al. (1998) to focus on cryptochrome (cry), a close homologue of the genes for blue-light receptors found in plants. When flies experienced normal light-dark cycles, CRY levels oscillate (albeit out of sync with those of PER and TIM), but when flies were kept in total darkness oscillation stops and CRY levels continue to increase. This strongly supported a role for CRY in the entrainment process.

Most of the research I have related was conducted on Drosophila, but mammalian researchers were paying close attention and drew upon it as a guide to their own research once it was discovered that there is not just one but three homologues of per in the mouse-Perl, Per2, and Per3 (Zylka et al. 1998). Mammalian researchers found both commonalities and differences between flies and mammals. All three mammalian PERs oscillate, but they peak during the day rather than night. Moreover, unlike Drosophila PER, the concentrations of mammalian PERs increase as a result of light exposure. Two homologues of cryptochrome were also found in mice and they were initially thought to play a role in entrainment as cry does in Drosophila (Miyamoto and Sancar 1998). But this interpretation was soon challenged when it was discovered that mutants lacking both CRY1 and CRY2 exhibited no rhythmicity in total darkness. Mutants lacking only CRY1 exhibited accelerated free-running periodicity and those lacking only CRY2 demonstrated slowed periodicity (van der Horst et al. 1999). The further fact that mice lacking both CRY1 and CRY2 exhibited chronically elevated levels of mPerl transcript, indicated that CRY1 and CRY2 play a role in inhibiting mPerl expression (Vitaterna et al. 1999). Researchers concluded that the CRY1and CRY2 had supplanted TIM as the partner for PER in the mammalian oscillator (see Bechtel 2009, for discussion of the role of the assumption of conservation in linking research on Drosophila and mammals).

Research in this century has continued to unveil additional components such as $\mathrm{ROR} \alpha, \mathrm{REV}-\mathrm{ERB} \alpha, \mathrm{DEC} 1$ and DEC2, which function to modulate the behavior of clock components such as CLOCK and BMAL1 (Honma et al. 2002; Preitner et al. 2002). The gap in the entrainment process left when the cryptochromes were found to be constituents in the central oscillator in mammals was filled by the discovery of melanopsin, a previously unrecognized member of the opsin family of photoreceptors (Hattar et al. 2003). The clockworks in the circadian clock turned out to be genes and proteins, and reductionistic research in the 1990s and first years of this century successfully revealed the identity of many of the components of the clock and the operations each participates in. By any criterion, reductionism yielded 
immense gains in knowledge of how circadian rhythms are generated in a very short period.

\section{The Upward Path I: An Organized Network within SCN Cells}

Discovering parts and operations, however, is only part of the challenge in mechanistic research and may be the easier part of the task. Think of a child taking a mechanical watch apart. Assume the child is very careful and lays out each of the parts separately and explores the operations each can perform. At this point the child will have learned a great deal but is still likely to be quite befuddled as to how the watch tells time. Figuring that out requires determining how the parts and operations are organized and work together in the watch. In circadian research, knowing the identity of the genes and proteins and the individual operations of gene expression and protein binding to gene promoters does not reveal how the mechanism comprised of these parts and operations serves to maintain a regular and approximately $24 \mathrm{~h}$ oscillation that is temperature compensated and entrainable by environmental cues. Explaining these circadian phenomena required conceptualizing how the parts and operations are organized and orchestrated.

Figure 1 above already introduced a key organizing principle that provided the skeleton into which researchers could fit other components as they were identified. The discovery that TIM (in Drosophila) and CRY (in mammals) dimerized with PER made it easy to figure out how to incorporate them into the diagram. The determination that neither PER nor TIM could operate directly on its own DNA opened up a space to be filled by BMAL1 and CLOCK and it was determined that the BMAL1:CLOCK dimer performs this role by binding to the Ebox promoter on the Per and Cry genes. BMAL1 and CLOCK arise outside this negative feedback loop and serve as the activator of the process regulated by the feedback. Figure 2 shows these components, plus the roles played by ROR $\alpha$ and Rev-erb $\alpha$ in exciting and inhibiting the formation of Bmal1. Not shown is the role of Casein Kinase $\mathrm{I} \varepsilon$ and $\mathrm{I} \delta$ in phosphorylating PER. The figure is still highly simplified; it leaves out several known components and does not differentiate the multiple versions of Per and Cry.

Figure 2 provides a different understanding of the clock than that provided by knowing the identity of the individual genes and proteins since it focuses on how the components interact. But there is more to be understood at this level-the different operations occur at different rates at different times and so need to be orchestrated to maintain a $24 \mathrm{~h}$ oscillation. Understanding this requires simulating the coordinated operation of the parts. For simple mechanisms, researchers can carry out simulations in their heads. One could do that even with a complex interaction of components as in Fig. 2 if each operation was completed before the next began. But in this case different operations are occurring at the same time, and the rate at which each is performed varies across time. To understand the effects this can produce, researchers often appeal to computational simulations. This involves writing differential equations describing how the rates of individual operations are affected by various parameters and using computers to show how a system involving such 


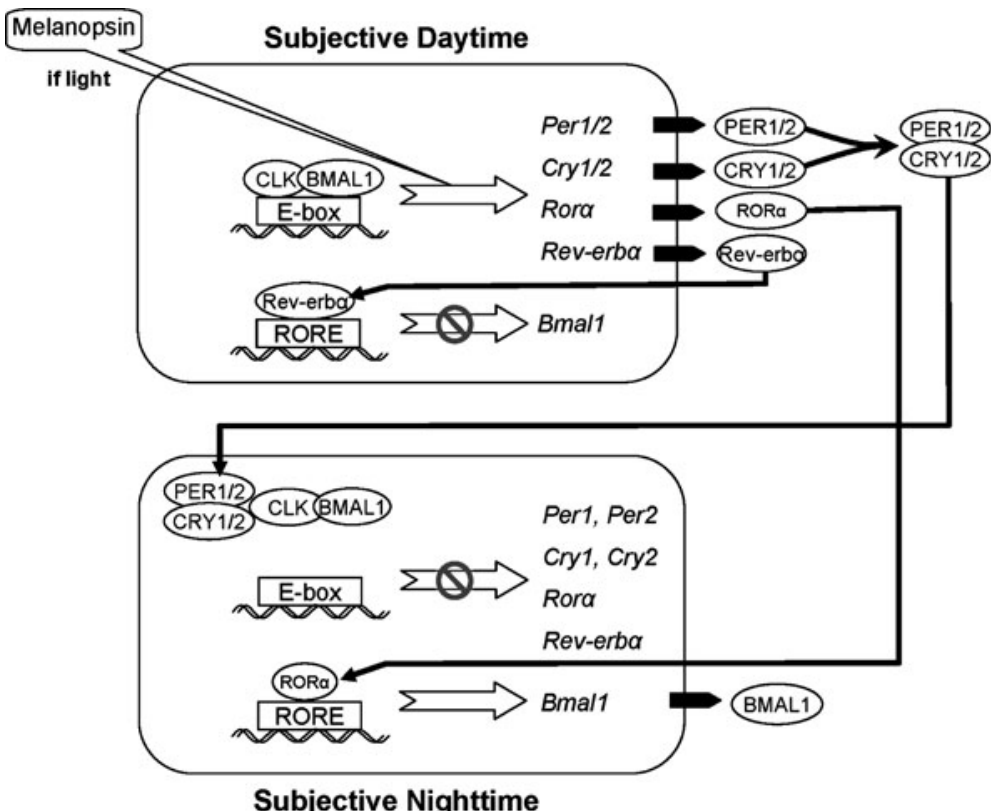

Fig. 2 A simplified diagram of the mammalian circadian oscillator

operations would change over time (and respond to various perturbations). Such simulations reveal whether the set of temporally characterized operations can account for the target behavior. Sometimes modelers try to pare their models down to a minimum of components needed to generate the phenomena in the interest of promoting explanatory understanding (Forger et al. 2007), while at other times they seek to provide as complete an account as possible, including all known components and their operations (see Leloup and Goldbeter 2003, for such a model of the mammalian circadian oscillator). Typically simulations of either sort succeed in accounting for some aspects of the phenomena but not others and then serve to guide further empirical research. Here I cannot consider further the role of modeling in mechanistic research (see Bechtel and Abrahamsen 2010, for further discussion), but only note its importance as a tool for moving up from the behavior of individual components to understanding their coordinated function in a system with complex interactions.

\section{The Upward Path II: Networks between Cells and between Organs}

So far I have focused on timekeeping as an intracellular activity, but increasingly mammalian researchers are recognizing the need to look at still higher system-level processing, both within the SCN and between the SCN and oscillators in other organs of the body. I consider first intercellular organization within the SCN. 
I noted earlier that the SCN on each side of the brain contains approximately 8,000-10,000 neurons. Given that individual cells maintain a circadian rhythm, one might wonder why there are so many cells. Are they simply redundant to each other? It is becoming increasingly clear that they are not. ${ }^{1}$ The cycling periods of individual cells differ rather dramatically from $24 \mathrm{~h}$. When Welsh et al. (1995) cultured cells on a microelectrode array (allowing recording from multiple cells that were oscillating independently), they found substantial variability between the rhythms maintained in individual cells: a standard deviation of $1.2 \mathrm{~h}$ and a range from 21.25 to $26.25 \mathrm{~h}$. Liu et al. (1997) went onto show that the circadian period in animal behavior corresponds to the average across a large number of individual SCN cells. Moreover, when spatial relations between SCN neurons are maintained in explants, the variability between cells was much reduced, indicating that oscillations in individual neurons were being synchronized to those in others (Herzog et al. 2004). (For a discussion of computational modeling of such synchronization, see Beersma 2005). Recent studies point to vasoactive intestinal polypeptide (VIP) as a major agent for establishing synchrony, although GABA and gap junctions may also be involved (Vansteensel et al. 2008). In addition, Liu et al. (2007) found that in the SCN the interactions between cells are able to compensate for genetic losses (e.g., loss of Cryl) that are sufficient to disrupt rhythms either in isolated SCN cells or in peripheral oscillators. Their modeling efforts pointed to weak residual oscillations in some neurons that are then communicated to others to generate the global response. These findings indicate that individual SCN neurons are not just redundant timekeepers but exist in a network which as a whole exhibits reliable timekeeping. Liu et al. (p. 612) comment: "the SCN circadian clock is more than just the sum of its cells."

Important connections that influence circadian behavior, though, extend beyond the SCN proper. Figure 3 depicts the framework that implicitly guided much clock research through the late twentieth century: signals from light, the primary Zeitgeber, are processed by photoreceptors, whose output entrains the central oscillator. The oscillator produces outputs which determine the behavior of peripheral clocks, which in turn regulate behavior. Increasingly, though, researchers are coming to regard this picture as problematic; they are exploring ways in which operations thought to be downstream of a given operation influence that upstream operation.

To begin with a simple example, the ability of receptors to detect light is affected by the behavior of the organism (e.g., if it burrows in the sand or comes out of a cave) and if that behavior is under circadian control, then so is the processing by its photoreceptors. Sometimes even the response properties of the receptors are altered on a circadian basis. The unicellar organism Gonyaulax polyedra has two circadian photoreceptor systems, and the one responsive to blue light (which results in large advances in circadian periods) oscillates under circadian control (Roenneberg and Deng 1997). Similar circadian control over the entrainment photoreceptors have

\footnotetext{
1 There is not space in this paper to consider the differences between regions in the SCN although there is evidence that different parts of the SCN behave differently and respond on different timescales to light entrainment (Nakamura et al. 2005).
} 


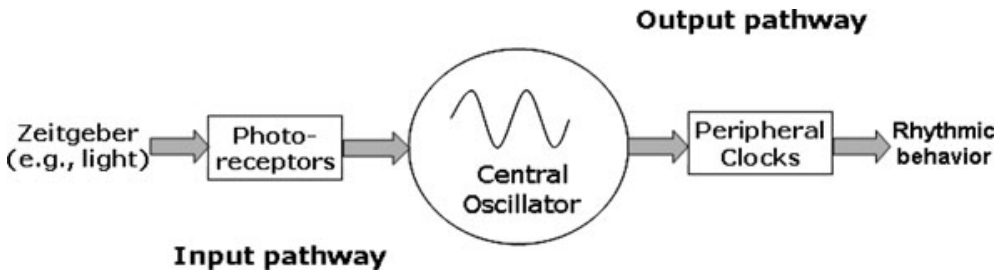

Fig. 3 Classical feedforward view of the circadian system

been identified in plants (Millar 2003) and some investigators think that melanopsin, the photoreceptor in retinal ganglion cells, provides the input signal for entraining the SCN oscillators, is also under circadian control (Rollag et al. 2003). Results such as these led Roenneberg et al. (2003, p. 190) to conclude that "the clock changes the properties of the input." To reflect this, a feedback arrow from the central oscillator to the photoreceptors has been included in Fig. 4.

It has long been thought that there are clocks in peripheral tissues (in addition to the central SCN oscillator), but until recently they were assumed to be few in number and passively driven by (slaves to) the SCN. The slave view was supported by the early research showing that lesions to the SCN alone generated behavioral arrhythmia and more recent studies showing that peripheral oscillators do not appear to be able to sustain themselves (Yamazaki et al. 2000) and that normal rhythms could be restored when peripheral oscillators extracted form mice with mutant rhythms were implanted in an organism with a normally functioning SCN (Pando et al. 2002).

Both parts of this view have subsequently been contested. Key clock proteins were found to oscillate in a wide range of tissue types in Drosophila (Hardin 1994; Plautz et al. 1997). In their study showing three homologues of PER in mammals, Zylka et al. (1998) also found that these proteins cycled in various peripheral tissues, including other regions of the brain and in liver, skeletal muscle, and testis. These and subsequent results revealing that in many tissues nearly $10 \%$ of gene products cycle (different ones in different tissues) led Schibler and Sassone-Corsi (2002, p. 919) to speculate that the "circadian timing system is composed of almost as many individual clocks as there are cells." The assumption that they were

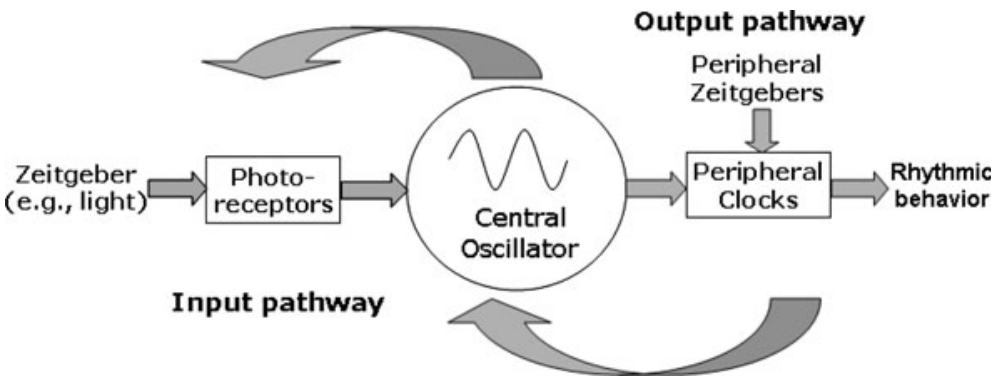

Fig. 4 Feedforward model supplemented by feedback loops and separate inputs to the peripheral clocks 
passively driven by the SCN was first challenged by the discovery that mammalian fibroblasts in long-term culture could sustain a circadian rhythm following a serum shock (Balsalobre et al. 1998). Subsequently, use of a luciferase Per2 fusion protein has revealed that oscillations are sustained for over twenty cycles in peripheral tissues in mammals (Yoo et al. 2004). The apparent dampening is due to deschronization between cells in peripheral oscillators, which has the effect of blurring the rhythms of individual oscillators (Welsh et al. 2004).

What does seem to be true is that the SCN is required to maintain synchrony between peripheral oscillators. Moreover, whereas the SCN responds to a new entrainment signal on the next cycle, peripheral oscillators adjust only after several complete daily cycles (Yamazaki et al. 2000; Davidson et al. 2008). The SCN thus performs a distinctive role in coordinating other clocks, but the other clocks are not merely responsive slaves. Accordingly, Davidson et al. (2004) reject the metaphor of SCN as ringmaster and proposed it be viewed as an orchestra conductor.

In non-mammalian species such as Drosophila and zebra-fish, peripheral clocks can be independently entrained by light (Giebultowicz 2001). Although this is not possible in mammals, some peripheral oscillators are entrained by chemical and temperature Zeitgebers (Balsalobre et al. 2000). Stokkan et al. (2001) maintained rats, which are usually nocturnal, in continuous darkness but fed them during their subjective day. Their liver oscillators, but not others, entrained to feeding, shifting their circadian rhythm forward $10 \mathrm{~h}$ in just 2 days, indicating a fair amount of independent behavior by peripheral oscillators. This raises the question whether peripheral clocks exert any influence back on the SCN. Recent studies using altered feeding regimes with blind mice have suggested that the SCN can in fact be entrained via feedbacks from the peripheral clocks (Castillo et al. 2004).

One of the clearest examples of feedback onto the central oscillator involves melatonin, a hormone synthesized in the pineal gland in vertebrates. In many nonmammalian vertebrates light penetrates to the pineal gland and it figures in the entrainment pathway. While this capacity has been lost in mammals, there is a processing loop from the SCN to the pineal gland and back. The pathway from the SCN to the pineal gland has been well studied and serves to shut down melatonin synthesis during the subjective day or in response to a light stimulus at night. When melatonin is synthesized, it is detected by two receptors ( $\mathrm{MT}_{1}$ and $\mathrm{MT}_{2}$ ) in the SCN, which then differentially mediate clock resetting at dusk and dawn (Hunt et al. 2001).

Another example of feedback from the periphery onto the SCN recently receiving attention involves circadian modulation of the mitochondrial oxidative phosphorylation pathway that affects oxidative-reduction potentials (redox) within cells. Redox potential in turn affects the concentrations of crucial oscillator components Clock, Mop3, and NPAS2 and thereby the rate of reactions within the central oscillator (Rutter et al. 2001). As Panda and Hogenesch (2004, p. 381) conclude, "these observations may indicate that genes involved in redox regulation are both outputs of the clock as well as feedback on clock function."

To accommodate findings such as these, separate inputs to the peripheral clocks and a second feedback arrow, this one from the peripheral clocks to the central oscillator, are included in Fig. 4. The two feedback arrows indicate that there is an 
integrated circadian system and that a step up to a systems level that considers interactions throughout the organism is needed to understand how circadian oscillators are entrained and influence other biological processes.

\section{Conclusions}

Reductionist research on circadian rhythms achieved tremendous success in the last decade of the twentieth century, resulting in identification of many of the crucial genes and proteins that constitute the clockworks of many species. This program, which continues today, was essential for advancing our understanding of circadian phenomena. For philosophy of science though, there is an additional lesson: as important as this reductionistic research was, its own discoveries exposed the need for complementary research at several levels of organization above the individual genes and proteins. Within individual SCN cells the various components are continually affected by the operations of the other components so that researchers must focus on the integration of the whole system to understand how cells maintain circadian oscillations. Reliable timing, though, only emerges from interactions of SCN cells whereby they synchronize with each other. Moreover, the SCN does not operate on its own but is situated between input photoreceptors and peripheral clocks linked to specific physiological and behavioral processes. There are feedback interactions between these that have sparked analysis at the systems level to complement reductionistic research on component parts and operations.

One of the virtues of thinking about reduction in the context of mechanisms (in contrast to more traditional theory-reduction accounts) is that the mechanistic account does not construe all the explanatory work as being done by the lowest level (Bechtel 2008). The analysis of circadian rhythms advanced here is compatible with Craver's (2007) mosaic, inter-level integration via multilevel mechanisms, but draws out a feature he does not emphasize - that the integration of lower-level components into mechanisms can be iterated so that a mechanism itself is part of a mechanism at a higher level. Craver and Bechtel (2007) have defended an account on which all entities that interact causally are at a single level, but on which lower levels affect higher levels, and vice versa, vis a vis the constitution relation that holds between mechanisms and their components. On this account, entities at different levels engage in different causal processes, and those at higher levels are not redundant (as Kim 1993, would suggest) to those at lower-levels. On our account, there is neither bottom-up nor top-down causation, but the phenomena these notions are intended to capture can be accommodated by combining intralevel causal relations at various levels and inter-level constitutive relations. The conclusion for which I am arguing here is that often the full causal account of a mechanism must include the causal processes at all levels or it will not be able to account fully for the phenomena. Accordingly, reductionist research must be complemented by investigations of the organization provided at higher levels to understand how mechanisms produce the phenomena for which explanation is sought. 
Acknowledgments I thank Adele Abrahamsen, John Bickle, Carl Craver, Robert Richardson, Karola Stotz, Paul Teller, David Welsh, and an anonymous referee for very helpful comments and discussion.

Open Access This article is distributed under the terms of the Creative Commons Attribution Noncommercial License which permits any noncommercial use, distribution, and reproduction in any medium, provided the original author(s) and source are credited.

\section{References}

Balsalobre, A., Brown, S. A., Marcacci, L., Tronche, F., Kellendonk, C., Reichardt, H. M., et al. (2000). Resetting of circadian time in peripheral tissues by glucocorticoid signaling. Science, 289(5488), 2344-2347.

Balsalobre, A., Damiola, F., \& Schibler, U. (1998). A serum shock induces circadian gene expression in mammalian tissue culture cells. Cell, 93(6), 929-937.

Bechtel, W. (2008). Mental mechanisms: Philosophical perspectives on cognitive neuroscience. London: Routledge.

Bechtel, W. (2009). Generalization and discovery through conserved mechanisms: Cross species research on circadian oscillators. Philosophy of Science, 76, 762-773.

Bechtel, W., \& Abrahamsen, A. (2010). Dynamic mechanistic explanation: Computational modeling of circadian rhythms as an exemplar for cognitive science. Studies in History and Philosophy of Science Part A.

Bechtel, W., \& Hamilton, A. (2007). Reduction, integration, and the unity of science: Natural, behavioral, and social sciences and the humanities. In T. Kuipers (Ed.), Philosophy of science: Focal issues. New York: Elsevier.

Bechtel, W., \& Richardson, R. C. (1993). Discovering complexity: Decomposition and localization as strategies in scientific research. Princeton, NJ: Princeton University Press.

Beersma, D. G. M. (2005). Why and how do we model circadian rhythms? Journal of Biological Rhythms, 20(4), 304-313.

Bickle, J. (2003). Philosophy and neuroscience: A ruthlessly reductive account. Dordrecht: Kluwer.

Castillo, M. R., Hochstetler, K. J., Tavernier, R. J., Jr., Greene, D. M., \& Bult-Ito, A. (2004). Entrainment of the master circadian clock by scheduled feeding. American Journal of Physiology: Regulatory, Integrative and Comparative Physiology, 287(3), R551-R555.

Craver, C. (2007). Explaining the brain: What a science of the mind-brain could be. New York: Oxford University Press.

Craver, C., \& Bechtel, W. (2007). Top-down causation without top-down causes. Biology and Philosophy, 22, 547-563.

Darlington, T. K., Wager-Smith, K., Ceriani, M. F., Staknis, D., Gekakis, N., Steeves, T. D., et al. (1998). Closing the circadian loop: CLOCK-induced transcription of its own inhibitors per and tim. Science, 280(5369), 1599-1603.

Davidson, A. J., Castanon-Cervantes, O., Leise, T. L., Molyneux, P. C., \& Harrington, M. E. (2008). Visualizing jet lag in the mouse suprachiasmatic nucleus and peripheral circadian timing system. European Journal of Neuroscience, 9999(9999).

Davidson, A. J., Yamazaki, S., \& Menaker, M. (2004). SCN: Ringmaster of the circadian circus or conductor of the circadian orchestra? In D. J. Chadwick \& J. A. Goode (Eds.), Molecular clocks and light signalling (pp. 110-125). Chichester, UK: John Wiley.

Emery, P. T., So, W. V., Kanedo, M., Hall, J. C., \& Rosbash, M. (1998). CRY, a Drosophila clock and light-regulated cryptochrome, is a major contributor to circadian rhythm resetting and photosensitivity. Cell, 95, 669-679.

Forger, D., Gonze, D., Virshup, D., \& Welsh, D. K. (2007). Beyond intuitive modeling: Combining biophysical models with innovative experiments to move the circadian clock field forward. Journal of Biological Rhythms, 22, 200-210.

Giebultowicz, J. M. (2001). Peripheral clocks and their role in circadian timing: Insights from insects. Philosophical Transactions of the Royal Society of London. B. Biological Sciences, 356, 1791-1799.

Hardin, P. E. (1994). Analysis of clock proteins in mouse SCN demonstrates phylogenetic divergence of the circadian clockwork and resetting mechanisms. Molecular and Cellular Biology, 14, 7211-7218. 
Hardin, P. E., Hall, J. C., \& Rosbash, M. (1990). Feedback of the Drosophila period gene product on circadian cycling of its messenger RNA levels. Nature, 343(6258), 536-540.

Hattar, S., Lucas, R. J., Mrosovsky, N., Thompson, S., Douglas, R. H., Hankins, M. W., et al. (2003). Melanopsin and rod-cone photoreceptive systems account for all major accessory visual functions in mice. Nature, 424(6944), 75-81.

Herzog, E. D., Aton, S. J., Numano, R., Sakaki, Y., \& Tei, H. (2004). Temporal precision in the mammalian circadian system: A reliable clock from less reliable neurons. Journal of Biological Rhythms, 19(1), 35-46.

Honma, S., Kawamoto, T., Takagi, Y., Fujimoto, K., Sato, F., Noshiro, M., et al. (2002). Dec1 and Dec2 are regulators of the mammalian molecular clock. Nature, 419(6909), 841-844.

Huang, Z. J., Edery, I., \& Rosbash, M. (1993). PAS is a dimerization domain common to Drosophila period and several transcription factors. Nature, 364(6434), 259-262.

Hunt, A. E., Al-Ghoul, W. M., Gillette, M. U., \& Dubocovich, M. L. (2001). Activation of MT2 melatonin receptors in rat suprachiasmatic nucleus phase advances the circadian clock. American Journal of Physiology: Cell Physiology, 280(1), C110-C118.

Hunter-Ensor, M., Ousley, A., \& Sehgal, A. (1996). Regulation of the Drosophila protein timeless suggests a mechanism for resetting the circadian clock by light. Cell, 84(5), 677-685.

Inouye, S.-I. T., \& Kawamura, H. (1979). Persistence of circadian rhythmicity in a mammalian hypothalamic "island" containing the suprachiasmatic nucleus. Proceedings of the National Academy of Sciences (USA), 76, 5962-5966.

Kim, J. (1993). Supervenience and the mind. Cambridge: Cambridge University Press.

Konopka, R. J., \& Benzer, S. (1971). Clock mutants of Drosophila melanogaster. Proceedings of the National Academy of Sciences (USA), 89, 2112-2116.

Leloup, J.-C., \& Goldbeter, A. (2003). Toward a detailed computational model for the mammalian circadian clock. Proceedings of the National Academy of Sciences, 100(12), 7051-7056.

Liu, X., Lorenz, L., Yu, Q. N., Hall, J. C., \& Rosbash, M. (1988). Spatial and temporal expression of the period gene in Drosophila melanogaster. Genes and Development, 2, 228-238.

Liu, C., Weaver, D. R., Strogatz, S. H., \& Reppert, S. M. (1997). Cellular construction of a circadian clock: Period determination in the suprachiasmatic nuclei. Cell, 91(6), 855-860.

Liu, A. C., Welsh, D. K., Ko, C. H., Tran, H. G., Zhang, E. E., Priest, A. A., et al. (2007). Intercellular coupling confers robustness against mutations in the SCN circadian clock network. Cell, 129(3), 605-616.

Machamer, P., Darden, L., \& Craver, C. (2000). Thinking about mechanisms. Philosophy of Science, 67, $1-25$.

Millar, A. J. (2003). A suite of photoreceptors entrains the plant circadian clock. Journal of Biological Rhythms, 18(3), 217-226.

Miyamoto, Y., \& Sancar, A. (1998). Vitamin B2-based blue-light photoreceptors in the retinohypothalamic tract as the photoactive pigments for setting the circadian clock in mammals. Proceedings of the National Academy of Sciences, 95(11), 6097-6102.

Moore, R. Y. (1973). Retinohypothalamic projection in mammals: A comparative study. Brain Research, 49, 403-409.

Moore, R. Y., \& Eichler, V. B. (1972). Loss of a circadian adrenal corticosterone rhythm following suprachiasmatic lesions in the rat. Brain Research, 42, 201-206.

Nagel, E. (1961). The structure of science. New York: Harcourt, Brace.

Nakamura, W., Yamazaki, S., Takasu, N. N., Mishima, K., \& Block, G. D. (2005). Differential response of Period 1 expression within the suprachiasmatic nucleus. Journal of Neuroscience, 25(23), 5481-5487.

Panda, S., \& Hogenesch, J. B. (2004). It's all in the timing: many clocks, many outputs. Journal of Biological Rhythms, 19(5), 374-387.

Pando, M. P., Morse, D., Cermakian, N., \& Sassone-Corsi, P. (2002). Phenotypic rescue of a peripheral clock genetic defect via SCN hierarchical dominance. Cell, 110(1), 107-117.

Plautz, J. D., Kaneko, M., Hall, J. C., \& Kay, S. A. (1997). Independent photoreceptive circadian clocks throughout Drosophila. Science, 278(5343), 1632-1635.

Preitner, N., Damiola, F., Luis Lopez, M., Zakany, J., Duboule, D., Albrecht, U., et al. (2002). The orphan nuclear receptor REV-ERB $\alpha$ controls circadian transcription within the positive limb of the mammalian circadian oscillator. Cell, 110(2), 251-260.

Price, J. L., Blau, J., Rothenfluh, A., Abodeely, M., Kloss, B., \& Young, M. W. (1998). Double-time is a novel Drosophila clock gene that regulates PERIOD protein accumulation. Cell, 94, 83-95. 
Ralph, M. R., Foster, R. G., Davis, F. C., \& Menaker, M. (1990). Transplanted suprachiasmatic nucleus determines circadian period. Science, 247(4945), 975-978.

Richter, C. P. (1967). Sleep and activity: Their relation to the 24 h clock. In S. S. Kety, E. V. Evarts, \& H. L. Williams (Eds.), Sleep and altered states of consciousness (pp. 8-29). Baltimore: Williams and Wilkins.

Roenneberg, T., Daan, S., \& Merrow, M. (2003). The art of entrainment. Journal of Biological Rhythms, 18(3), 183-194.

Roenneberg, T., \& Deng, T.-S. (1997). Photobiology of the Gonyaulax circadian system. I. Different phase response curves for red and blue light. Planta, 2002(4), 491-501.

Rollag, M. D., Berson, D. M., \& Provencio, I. (2003). Melanopsin, ganglion-cell photoreceptors, and mammalian photoentrainment. Journal of Biological Rhythms, 18(3), 227-234.

Rosenberg, A. (2006). Darwinian reductionism, Or, how to stop worrying and love molecular biology. Chicago: University of Chicago Press.

Rutter, J., Reick, M., Wu, L. C., \& McKnight, S. L. (2001). Regulation of Clock and NPAS2 DNA binding by the redox state of NAD cofactors. Science, 293(5529), 510-514.

Saez, L., \& Young, M. W. (1996). Regulation of nuclear entry of the Drosophila-clock proteins period and timeless. Neuron, 17, 979-990.

Schibler, U., \& Sassone-Corsi, P. (2002). A web of circadian pacemakers. Cell, 111(7), 919-922.

Sehgal, A., Price, J. L., Man, B., \& Young, M. W. (1994). Loss of circadian behavioral rhythms and per RNA oscillations in the Drosophila mutant timeless. Science, 263, 1603-1606.

Stephan, F. K., \& Zucker, I. (1972). Circadian rhythms in drinking behavior and locomotor activity of rats are eliminated by hypothalamic lesions. Proceedings of the National Academy of Sciences (USA), 69, 1583-1586.

Stokkan, K.-A., Yamazaki, S., Tei, H., Sakaki, Y., \& Menaker, M. (2001). Entrainment of the circadian clock in the liver by feeding. Science, 291(5503), 490-493.

Thagard, P. (2006). Hot thought: Mechanisms and applications of emotional cognition. Cambridge, MA: MIT Press.

van der Horst, G. T. J., Muijtjens, M., Kobayashi, K., Takano, R., Kanno, S.-I., Takao, M., et al. (1999). Mammalian Cry1 and Cry2 are essential for maintenance of circadian rhythms. Nature, 398(6728), 627-630.

Vansteensel, M. J., Michel, S., \& Meijer, J. H. (2008). Organization of cell and tissue circadian pacemakers: A comparison among species. Brain Research Reviews, 58(1), 18-47.

Vitaterna, M. H., King, D. P., Chang, A.-M., Kornhauser, J. M., Lowrey, P. L., McDonald, J. D., et al. (1994). Mutagenesis and mapping of a mouse gene, clock, essential for circadian behavior. Science, 264(5159), 719-725.

Vitaterna, M. H., Selby, C. P., Todo, T., Niwa, H., Thompson, C., Fruechte, E. M., et al. (1999). Differential regulation of mammalian period genes and circadian rhythmicity by cryptochromes 1 and 2. Proceedings of the National Academy of Sciences, 96(21), 12114-12119.

Vosshall, L. B., Price, J. L., Sehgal, A., Saez, L., \& Young, M. W. (1994). Block in nuclear localization of period protein by a second clock mutation, timeless. Science, 263(5153), 1606-1609.

Welsh, D. K., Logothetis, D. E., Meister, M., \& Reppert, S. M. (1995). Individual neurons dissociated from rat suprachiasmatic nucleus express independently phased circadian firing rhythms. Neuron, 14(4), 697-706.

Welsh, D. K., Yoo, S.-H., Liu, A. C., Takahashi, J. S., \& Kay, S. A. (2004). Bioluminescence imaging of individual fibroblasts reveals persistent, independently phased circadian rhythms of clock gene expression. Current Biology, 14(24), 2289-2295.

Yamazaki, S., Numano, R., Abe, M., Hida, A., Takahashi, R.-I., Ueda, M., et al. (2000). Resetting central and peripheral circadian oscillators in transgenic rats. Science, 288(5466), 682-685.

Yoo, S.-H., Yamazaki, S., Lowrey, P. L., Shimomura, K., Ko, C. H., Buhr, E. D., et al. (2004). PERIOD2:LUCIFERASE real-time reporting of circadian dynamics reveals persistent circadian oscillations in mouse peripheral tissues. Proceedings of the National Academy of Sciences, 101(15), 5339-5346.

Zylka, M. J., Shearman, L. P., Weaver, D. R., \& Reppert, S. M. (1998). Three period homologs in mammals: Differential light responses in the suprachiasmatic circadian clock and oscillating transcripts outside of brain. Neuron, 20(6), 1103-1110. 